\title{
Research Paper: Lentiviral Mediated Expression of Soluble Neuropilin 1 Inhibits Semaphorin 3A-mediated Collapse Activity in Vitro
}

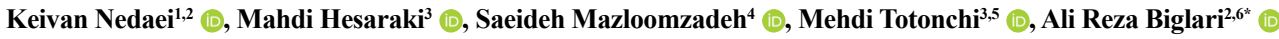 \\ 1. Department of Medical Biotechnology, School of Medicine, Zanjan University of Medical Sciences, Zanjan, Iran. \\ 2. Cancer Gene Therapy Research Center, Zanjan University of Medical Sciences, Zanjan, Iran. \\ 3. Department of Stem Cells and Developmental Biology, Cell Science Research Center, Royan Institute for Stem Cell Biology and Technology, \\ ACECR, Tehran, Iran. \\ 4. Social Determinants of Health Research Center, Zanjan University of Medical Sciences, Zanjan, Iran. \\ 5. Department of Genetics, Reproductive Biomedicine Research Center, Royan Institute for Reproductive Biomedicine, ACECR, Tehran, Iran \\ 6. Department of Genetics and Molecular Medicine, School of Medicine, Zanjan University of Medical Sciences, Zanjan, Iran.
}

\begin{tabular}{|c|c|}
\hline \multirow{2}{*}{$\begin{array}{l}\text { Us your device tos can } \\
\text { and read the article online }\end{array}$} & \\
\hline & Citation: Nedaei, K., Hesaraki, M., Mazloomzadeh, S., Totonchi, M., \& Biglari, A. (2021). Lentiviral Mediated Expression of \\
\hline 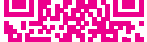 & Soluble Neuropilin 1 Inhibits Semaphorin 3A-mediated Collapse Activity in Vitro. Basic and Clinical Neuroscience, 12(2), 223- \\
\hline tepsit & dx.doi.org/10.32598/bcn.12.2.1678.1 \\
\hline 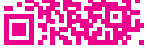 & doi http://dx.doi.org/10.32598/ben.12.2.1678.1 \\
\hline
\end{tabular}

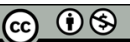

Article info:

Received: 15 Jan 2020

First Revision: 14 Mar 2020

Accepted: 02 Aug 2020

Available Online: 01 Mar 2021

Keywords:

Multiple sclerosis,

Neuropilin-1, Semaphorin 3A,

Remyelination

\section{A B S T RA C T}

Introduction: Semaphorin 3A (Sema 3A) is a secreted protein, which plays an integral part in developing the nervous system. It has collapse activity on the growth cone of dorsal root ganglia. After the development of the nervous system, Sema 3A expression decreases. Neuropilin 1 is a membrane receptor of Sema 3A. When semaphorin binds to neuropilin 1, the recruitment of oligodendrocyte precursor cells to the demyelinated site decreases. In Multiple Sclerosis (MS), Sema 3A expression increases and inhibits oligodendrocyte precursor cell differentiation. Therefore, the remyelination of axons gets impaired. We hypothesized that the function of Sema 3A could be inhibited by neutralizing its binding to membrane NRP1.

Methods: we cloned a soluble form of mouse Neuropilin 1 (msNRP1) in a lentiviral vector and expressed the recombinant protein in HEK293T cells. Then, the conditioned medium of the transduced cells was used to evaluate the effects of the msNRP1 on the inhibition of Sema 3A-induced growth cone collapse activity. Dorsal root ganglion explants of timed pregnant (E13) mice were prepared. Then, the growth cone collapse activity of Sema 3A was assessed in the presence and absence of msNRP1-containing conditioned media of transduced and non-transduced HEK293T cells. Comparisons between groups were performed by 1-way ANOVA and post hoc Tukey tests.

Results: msNRP1 was successfully cloned and transduced in HEK293T cells. The supernatant of transduced cells was concentrated and evaluated for the production of msNRP1. ELISA results indicated that transduced cells secreted msNRP1. Growth cone collapse assay showed that Sema 3A activity was significantly reduced in the presence of the conditioned medium of msNRP1-transduced HEK293T cells. Conversely, a conditioned medium of non-transduced HEK293T cells could not effectively prevent Sema 3A growth cone collapse activity.

Conclusion: Our results indicated that msNRP1 was successfully produced in HEK293T cells. The secreted msNRP1 effectively prevented Sema 3 A collapse activity. Therefore, msNRP1 can increase remyelination in MS lesions, although more studies using animal models are required. 


\section{Highlights}

- A soluble form of mouse Neuropilin1 (msNRP1) was cloned in a lentiviral vector.

- msNRP1 was successfully secreted by the transduced HEK293T in the supernatant.

- msNRP1-containing supernatant inhibited growth cone collapse activity of semaphorins 3A.

\section{Plain Language Summary}

Multiple Sclerosis (MS) is an inflammatory disease of the central nervous system. In MS, axons of neuronal cells become demyelinated, and the remyelination process is also defective. Oligodendrocyte precursor cells must survive, proliferate, and migrate towards demyelinated neurons and differentiate into mature oligodendrocytes to produce myelin and repair the neurons. Semaphorin 3A (Sema 3A) is a molecule that reduces the remyelination by preventing oligodendrocyte precursor cells from proliferating, migrating towards lesions, and differentiating into mature oligodendrocytes. We successfully produced mouse soluble Neuropilin 1 (msNRP1) that can bind Sema 3A and inhibit its activity. In other words, the msNRP1 was used to inhibit the activity of Sema 3A. Neural cells of dorsal root ganglia were cultured in the presence of Sema 3A with and without msNRP1. We observed that when msNRP1 was added to the neuronal cells culture, the growth cones could form. On the other hand, the growth cones collapsed when msNRP1 was not added to the cultures. This finding is promising in outcome of MS treatment. Remyelination is defective in MS patients, and axons are progressively demyelinated. Finding a molecule that can prevent demyelination and enhance remyelination will be of great importance for MS treatment and improvement of their condition.

\section{Introduction}

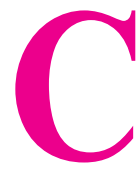

lass 3 semaphorins (Sema 3s) are secreted proteins in vertebrates. There are seven members of semaphorins (Sema 3A to Sema 3G), which have pivotal roles in nervous system development (Montolio et al., 2009). Semaphorin $3 \mathrm{~A}$ (Sema 3A) is the prototype of this class. It was isolated from the chicken brain for the first time and named collapsin 1, since it had collapse activity on Dorsal Root Ganglion (DRG) growth cones (Luo, Raible, \& Raper, 1993). Neurons of both the Peripheral Nervous System (PNS) and the Central Nervous System (CNS) express Sema 3A during the nervous system development. Sema $3 \mathrm{~A}$ becomes less abundant and more confined to particular regions of adults CNS. However, its expression increases in CNS conditions (De Winter et al., 2002; Good et al., 2004; Joyal Et Al., 2011; Körner et al., 2016) such as Multiple Sclerosis (MS) (Costa et al., 2015; Williams et al., 2007) as it was shown in animal models and human samples.

MS is an inflammatory demyelinating disease of the CNS. To remyelinate axons in MS, Oligodendrocyte Precursor Cells (OPCs) must survive, proliferate, migrate to lesions and differentiate into mature oligodendrocytes that produce myelin (Reviewed by Franklin, 2008). Remyelination defects might exist at any step in this process. Sema 3A amount increases in grey matter neurons, astrocytes, and microglial cells that surround or reside in active inflammatory lesions in MS patients and experimental models of demyelination (Williams et al., 2007). Research studies have demonstrated that Sema $3 \mathrm{~A}$ inhibits the recruitment of oligodendrocyte precursor cells to the demyelinated site (Piaton et al., 2011). It also prevents the differentiation of OPCs to myelinating oligodendrocyte cells (Syed et al., 2011).

On the other hand, Sema 3A disrupts the interaction of PP2A serine/threonine phosphatase and VE-cadherin in endothelial cells. This disruption can weaken tight junctions and increase vascular and Bood-Brain Barrier (BBB) permeability (Le Guelte et al., 2012). Increased permeability of BBB facilitates the access of immune system components to CNS. This, in turn, results in disease severity and progression. Therefore, Sema $3 \mathrm{~A}$ acts as a Vascular Endothelial Growth Factor (VEGF) in MS pathology. Sema 3A signaling is mediated by two important molecules: (i) neuropilin-1 as binding receptor and (ii) plexins as signal-transducing receptors.

Neuropilin-1 (NRP1) is a type-1 membrane glycoprotein. It also has four soluble forms in human (Cackowski, Xu, Hu, \& Cheng, 2004; Rossignol, Gagnon, \& Klagsbrun, 2000). The membrane form has a short cytoplasmic domain and an extracellular component. The 
former has no apparent signaling motif. The latter consists of $\mathrm{a} 1 / \mathrm{a} 2, \mathrm{~b} 1 / \mathrm{b} 2$, and $\mathrm{c}$ domains. The a1/a2 domain bind to Sema 3, and the b1/b2 domains bind to Sema 3 and VEGF (Miao et al., 1999). In transgenic mice, NRP1 overexpression results in embryonic lethality, neuronal guidance defects, and vascular formation (Kitsukawa, Shimono, Kawakami, Kondoh, \& Fujisawa, 1995). NRP1 disruption in mice is also embryonic lethal due to defective neuronal patterning and inadequate vascularization (Kitsukawa et al., 1997). The soluble isoforms have conserved extracellular domains. These domains are ligand-binding domains. However, c-domain, transmembrane, and intracellular domains are lacking. Panigrahy et al. cloned a mouse hepatic sNRP1 isoform. Their experiments showed that this endogenous inhibitor of angiogenesis might regulate VEGF or hepatocyte growth factor bioavailability during normal growth and development (Panigrahy et al., 2014). Besides, Gagnon et al. studied human soluble s11NRP1. They showed that s11NRP1 antagonized VEGF binding and consequently induced apoptosis in tumor cells. Their data suggested that soluble NRP1 and membrane-bound NRP1 have opposite functions (Gagnon et al., 2000).

Gene therapy is one of the leading areas of MS research. A suitable protein for MS gene therapy should have two characteristics: (i) it should induce axons remyelination by OPCs recruitment/differentiation (ii) it should reduce $\mathrm{BBB}$ permeability to restrict immune system access to the CNS. Therefore, in the present study, we investigated the inhibition of semaphorin $3 \mathrm{~A}$ growth cone collapse activity by mouse soluble neuropilin-1 in vitro.

\section{Methods}

\subsection{Lentiviral vector construction}

A murine soluble Neuropilin 1 (msNRP1)-expressing lentiviral vector (Lv-SFFV-msNRP1) was constructed based on the LeGO-iG2 plasmid (Weber, Bartsch, Stocking, \& Fehse, 2008) from Addgene. The msNRP1-coding sequence fragment was reverse transcribed from total RNA extracted from C57BL/6 mouse liver tissue. Subsequently, specific forward (F:5' GCTGAATTCATGGAGAGGGGGCTGCCGTT 3') and reverse primers (R:5' AGCAGGCCTCAGATAAGTATGTGAGCCCTTGTGC $3^{\prime}$ ) containing the restriction site for EcoR I and Stu I enzymes were used to amplify the fragment. The amplified DNA fragments were purified from agarose gel and cloned into a pLeGO-iG2, downstream of the Spleen Focus Forming Virus (SFFV) promoter. The recombinant plasmid was verified using PCR-Sanger sequencing as well as restriction digestion analysis.
For lentiviral vector packaging, HEK293T cells were transfected with pLeGO-SFFV/msNRP1-IRES-EGFP or pLeGO-SFFV/IRES-EGFP(the control vector), psPAX2, and pMID2.G plasmids in the presence of Lipofectamine 2000 (Thermo Fischer Scientific, USA). Forty-eight $h$ after transfection, culture medium containing lentiviral particles (Lv-SFFV-msNRP1 and Lv-SFFV-eGFP) were collected, passed through a $0.45 \mu \mathrm{m}$, and concentrated by ultracentrifugation at $82000 \mathrm{~g}$ for 2 hours.

\subsection{Production of sNRP1 by HEK293T cells}

To produce sNRP1 from the sNRP1-lentiviral vectors, HEK293T cells were plated on T75 flasks and cultured at $10^{4} \mathrm{cell} / \mathrm{cm}^{2}$ density in complete Dulbecco's Modified Eagle Medium (DMEM) (INOCLON, Iran) containing 10\% FBS (Gibco, Thermo Fischer Scientific, USA), 1x Pen-Strep (INOCLON, Iran), and $25 \mathrm{mM}$ 4-(2-hydroxyethyl)-1-piperazineethanesulfonic acid (HEPES) (INOCLON, Iran). At 50\% to $60 \%$ confluence, the culture medium was replaced with fresh DMEM containing $5 \mu \mathrm{L} / \mathrm{mL}$ of concentrated LV-SFFV-msNRP1 particles, 5\% FBS, $8 \mu \mathrm{g} / \mathrm{mL}$ polybrene (Sigma-Aldrich, USA), and $25 \mathrm{mM}$ HEPES (INOCLON, Iran). In control flasks, the medium was supplemented with the LvSFFV-eGFP particles. Twenty-four hours after incubating the cells, the old medium was replaced with fresh DMEM containing 5\% Fetal Bovine Serum (FBS) and 25 mM HEPES.

Every $24 \mathrm{~h}$, the supernatant of the transduced and nontransduced cells were collected for $72 \mathrm{~h}$ and pooled after centrifugation at $800 \times \mathrm{g}$ for 5 minutes. The molecular mass of the murine sNRP1 is estimated as $50-90 \mathrm{kDa}$ (Mamluk, Klagsbrun, Detmar, \& Bielenberg, 2005). Therefore, supernatant samples were first concentrated on $50 \mathrm{kDa}$ MWCO Amicon Ultra-15 devices (Merck Millipore, Germany) through 15 minutes of centrifugation at $5000 \times \mathrm{g}$. Concentrated samples were then subjected to a second concentration step on a $100 \mathrm{kDa} \mathrm{MWCO}$ Amicon Ultra-4 device (Merck Millipore, Germany) and centrifuged for 10 minutes at $5000 \times \mathrm{g}$. The total concentration factor was estimated at 20 times. The flowthrough was aliquoted and kept at $-20^{\circ} \mathrm{C}$.

The levels of concentrated and non-concentrated sNRP1 secreted from HEK293T cells were measured using Mouse neuropilin-1 PicoKine ${ }^{\mathrm{TM}}$ ELISA Kit (Boster Biological Technology, China) according to the manufacturer's instructions. The differential absorbance reads of 450-570 $\mathrm{nm}$ on an automated Microplate Reader (BioTek Instruments, USA) analyzed with the Gen5 
software (BioTek Instruments, USA) was used to measure sNRP1 concentration.

\subsection{Dorsal root ganglion explants culture}

Dorsal Root Ganglion (DRG) explants were isolated and cultured according to the protocol described by Wang and Marquardt (Wang \& Marquardt, 2012) with slight modifications. Briefly, DRGs were isolated from timed pregnant (E13) mice under the sterile condition and plated on pre-coated coverslips with poly L-lysine (Sigma-Aldrich, USA) $(1 \mathrm{mg} / \mathrm{mL})$ and laminin (SigmaAldrich, USA) $(100 \mu \mathrm{g} / \mathrm{mL})$. The explants were grown for $20 \mathrm{~h}$ in a Defined Medium (DM), consisting of neurobasal medium (Gibco, Thermo Fischer Scientific, USA) supplemented with $2 \mathrm{mM}$ L-glutamine (INOCLON, Iran), 2x B-27 (Gibco, Thermo Fischer Scientific, USA), 1x Pen-Strep, and 10 ng/mL Nerve Growth Factor (NGF) (R\&D Systems, USA).

\subsection{Growth cone collapse assay}

To assess semaphorin 3A (Sema 3A) inhibition by SNRP1, we defined five study groups in which Dorsal Root Ganglia (DRG) explants were exposed to Conditioned Media (CM) supplemented or not with $100 \mathrm{ng} /$ mL Sema 3A (R\&D System, USA). Conditioned media was the concentrated cell culture supernatant (DMEM containing 5\% FBS and $25 \mathrm{mM}$ HEPES) of transduced HEK293T, as previously mentioned, supplemented with 2 mM L-glutamine, 2x B-27, and 1x Pen-Strep.

To do so, DRG explants were first washed with prewarmed Phosphate-buffered saline (PBS) (INOCLON, Iran) and then incubated with a pre-warmed CM supplemented or not with $100 \mathrm{ng} / \mathrm{mL}$ Sema $3 \mathrm{~A}$ for $30 \mathrm{~min}$ at $37^{\circ} \mathrm{C}$. Incubation of DRG explants with the Defined Media (DM) (without NGF) contained or not $100 \mathrm{ng} /$ $\mathrm{mL}$ Sema 3A (controls). Before adding to cultured DRG explants, CMs and DMs were incubated for $30 \mathrm{~min}$ at $37^{\circ} \mathrm{C}$ to facilitate binding Sema $3 \mathrm{~A}$ to sNRP1. DRG explants were then stained with Alexa Fluor 488 phalloidin (Thermo Fischer Scientific, USA) for $4 \mathrm{~h}$ at room temperature following fixation, permeabilization, and blocking according to the previously described method (Wang \& Marquardt, 2012). Coverslips containing DRG were mounted using ProLong Gold antifade reagent (Thermo Fischer Scientific, USA).

\subsection{Imaging and quantification}

Fluorescent images were captured on an upright BX51 fluorescent microscope (Olympus, Japan) equipped with cell $^{\wedge} \mathrm{A}$ Imaging software (Olympus, Japan). For the quantification of collapsed growth cones, 10 DRG explants from each experimental group (in which at least 40 single identifiable growth cone per DRG had been analyzed) were scored as collapsed (growth cones without lamellipodia or with $<2$ filopodia or bullet-shaped) and non-collapsed (growth cones with the presence of lamellipodia and or $\geq 2$ filopodia) as described by Kapfhammer, Xu, and Raper (2007). Data are presented as the percentage of the collapsed growth cones to the total number of scored growth cones per DRG.

\subsection{Statistical analysis}

Values were expressed as Mean $\pm \mathrm{SE}$, and comparisons were performed by 1-way ANOVA and post hoc Tukey tests. $\mathrm{P}<0.05$ were considered significant. All analyses were performed using the SPSS V. 22.

\section{Results}

\subsection{Secretion of Murine sNRP1 from Lv-SFFV- msNRP1 tansduced HEK293 cells}

Lentiviral vectors were used to engineer HEK293T cells for the continuous production of msNRP1. To transduce the cultured HEK293T, two recombinant lentiviral vectors expressing Green fluorescent protein (GFP) (Lv-SFFV-eGFP) or GFP and murine sNRP1 (Lv-SFFV-msNRP1) were used (Figure 1A). Forty-eight $\mathrm{h}$ post-transduction, the transduced cells were monitored under a fluorescent microscope for the expression of GFP. As shown in Figure 1B, approximately $70 \%$ of the transduced cells seemed to express GFP, compared to non-transduced cells. Transduction does not seem to affect the cell's morphology when examined under phasecontrast microscopy (Figure 1B). In parallel, the amount of msNRP1 in the supernatant of the transduced and non-transduced HEK293T cells was measured. Unlike the non-transduced and Lv-SFFV-1eGFP-transduced cells in which no msNRP1 signal was detected, almost $100 \mathrm{ng} / \mathrm{mL}$ msNRP1 has been seen in the supernatant of Lv-SFFV-msNRP1-transduced HEK293Ts (Figure 1C). These data indicate that constructed Lv-SFFV-msNRP1 vectors successfully transduced HEK293T cells for the effective expression and secretion of murine sNRP1.

3.2. sNRP1 ability to inhibit sema 3A-induced growth cone collapse activity

To investigate sNRP1 capability of inhibiting the Sema 3A-induced growth cone collapse, Dorsal Root Ganglion (DRG) explants from E13 mouse embryos were cultured and treated with Seam $3 \mathrm{~A}$ in the presence or absence of sNRP1. The study groups included DRGs treated with con- 
(A)
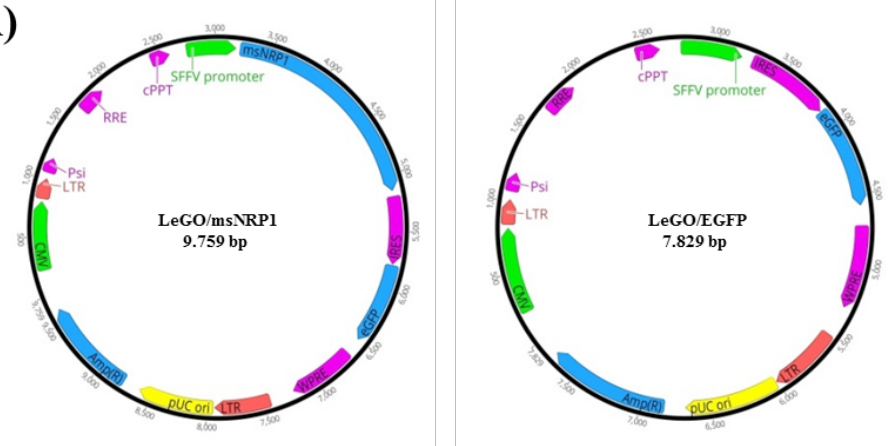

(B)
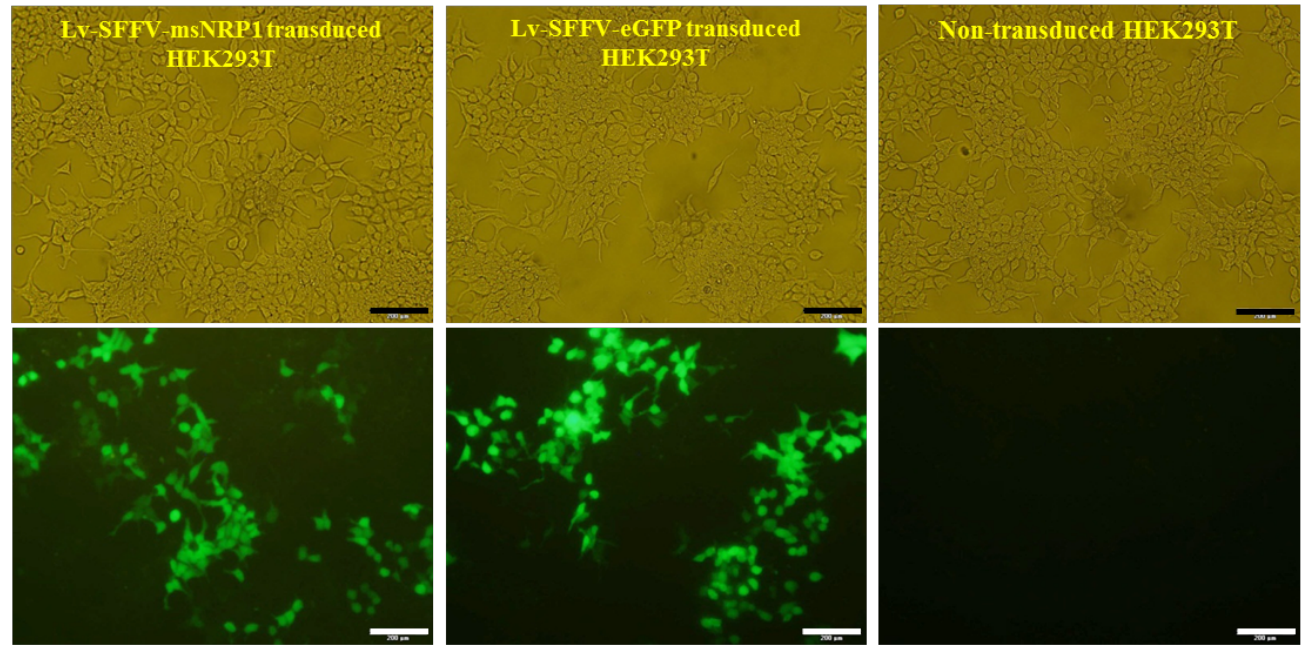

(C)

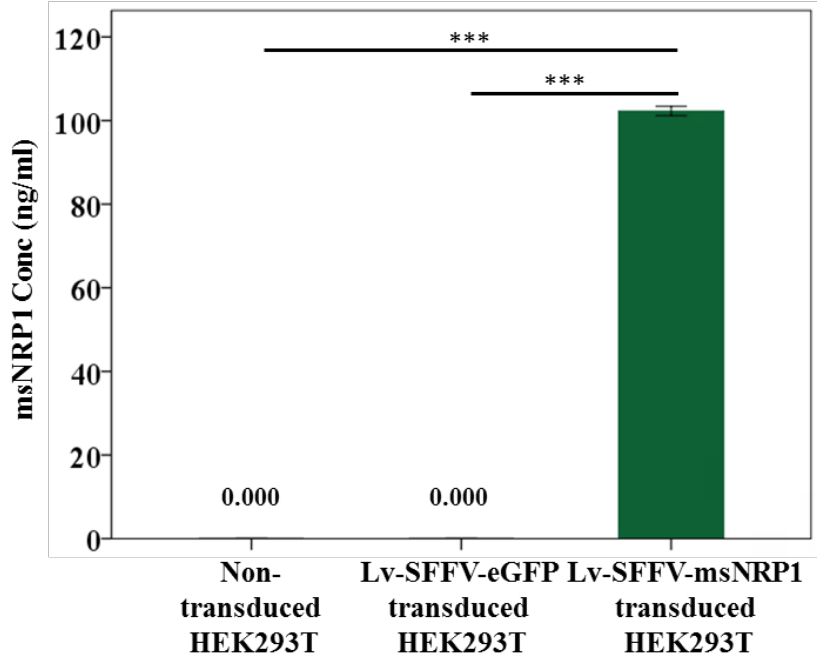

NEUROSCIENCE

Figure 1. Secretion of Murine sNRP1 from Lv-SFFV-msNRP1 transduced HEK293 cells

A: Lentiviral vectors used in this study. LeGO/msNRP1 (left) is the recombinant vector containing msNRP1 and GFP coding sequences; sNRP1 and GFP are connected by IRES sequence. Both coding sequences are under the control of a single SFFV promoter. LeGO/EGFP (right) is the control vector, which contains only GFP coding sequence; B: HEK293T cells after transfection. HEK293T cells transduced with LeGO/msNRP1 (left), LeGO/EGFP (middle), and non-transduced cells (right). GFP expression (green) indicates that HEK293T cells were successfully transduced with the lentiviral vectors (left and middle). Non-transduced cells do not show GFP expression (right); C: msNRP1 concentration in the supernatant of transduced and non-transduced cells. ELISA results indicated that the supernatant of non-transduced cells and Lv-SFFV-1eGFP-transduced cells had no detectable msNRP1. The supernatant of LvSFFV-msNRP1-transduced cells contained nearly $100 \mathrm{ng} / \mathrm{mL}$ of $\mathrm{msNRP1} ;{ }^{* * *} \mathrm{P}<0.001$ 
(A)

DM (without Sema 3A)

(B)

DM (+ Sema 3A)

(C)

\section{Non-transduced \\ HEK293T CM \\ (+ Sema 3A)}

(D)

Lv-SFFV-eGFP

transduced HEK293T

CM

(+ Sema 3A)

(E)

\section{Lv-SFFV-msNRP1} transduced HEK293T

\section{CM}

(+ Sema 3A)
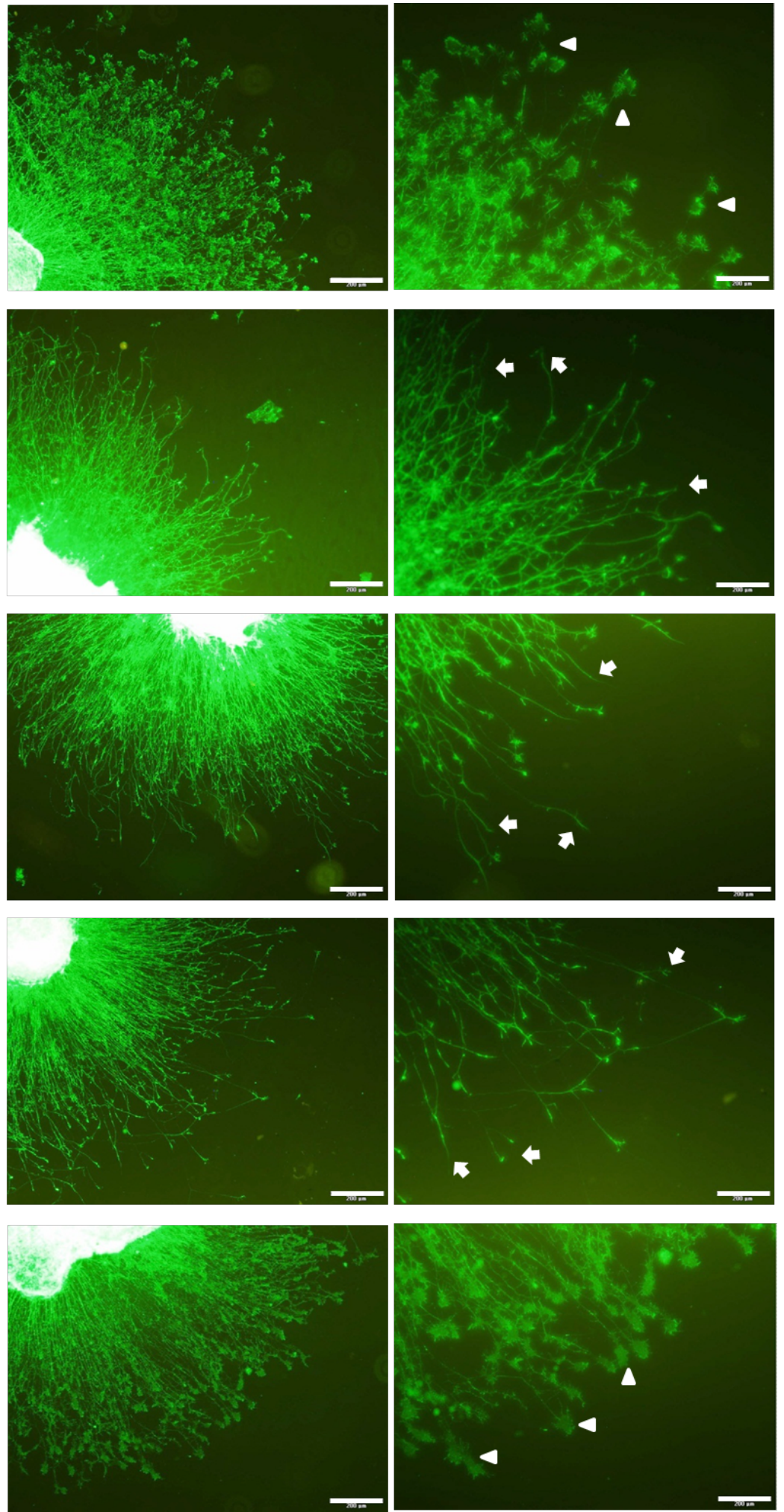

NEUR SCIENCE

Figure 2. Growth cone collapse activity assay

A and E: DRGs treated with DM without Sema 3A and CM + Sema 3A, respectively. Growth cones are not collapsed since Sema 3A was not added to DRGs (A), or msNRP1-containing CM inhibited Sema 3A binding to membrane NRP on DRGS. Arrowheads show the growth cones. B -D: DRGs treated with either Sema 3A-containing DM, Sema 3A-containing non-transduced cells' CM, or Sema 3Acontaining GFP-transduced cells' CM, respectively. The number of collapsed growth cones in B-D is significantly higher than that of A and B since there was no msNRP1 in the media to inhibit Sema 3A activity. Arrows show the collapsed growth cones. 


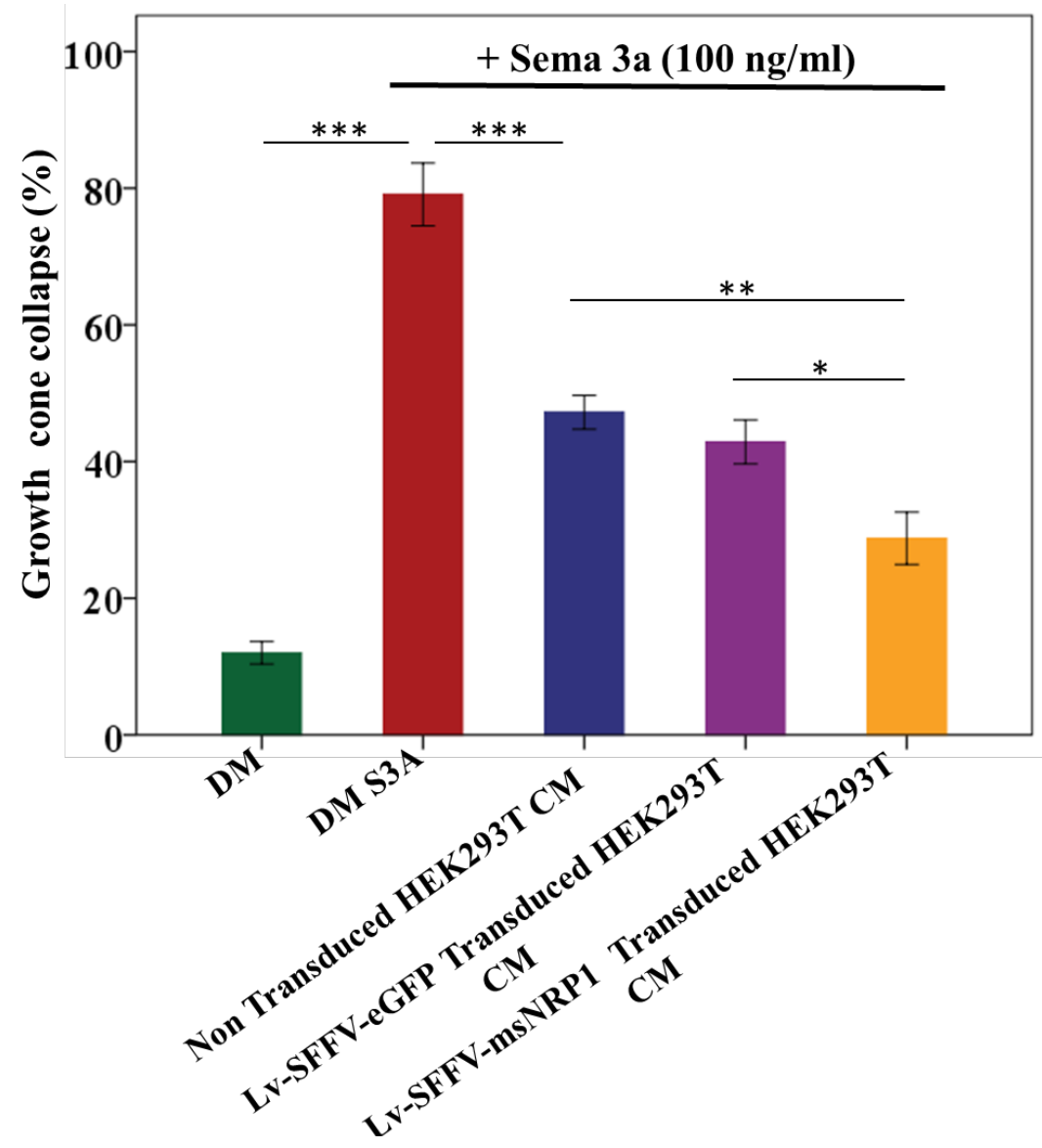

NEUR SCIENCE

Figure 3. Quantification of collapsed growth cones in the groups

Growth cone collapse was 12\% in DRGs treated with DM without Sema 3A, while 79\% collapsed growth cones were observed when DRGs were treated with Sema 3A-containing DM (DM S3A). Treatment of the DRGs with the CM of non-transduced cells, LeGO/ EGFP-transduced cells, and LeGO/sNRP1-transduced cells resulted in $47 \%, 43 \%$, and $27.7 \%$ growth cone collapse, respectively. ${ }^{* 2 *} \mathrm{P}<0.001,{ }^{*} \mathrm{P}<0.01,{ }^{*} \mathrm{P}<0.05$.

ditioned media from non-transduced and lentivirus vectors transduced HEK293T cells and defined media (with and without Sema 3A). Figure 2 shows fluorescent images of DRGs explants stained with Alexa Fluor 488 conjugated phalloidin to detect collapsed growth cones. The treatment of DRG with Sema 3A-supplemented supernatant of non-transduced and Lv-SFFV-eGFP vector-transduced HEK293T cells caused a large number of growth cones to be collapsed. On the other hand, the incubation of DRG with CM from Lv-SFFV-msNRP1 vector-transduced HEK293T led to the collapse of few numbers of growth cones compared to the above-mentioned groups (Figure 2).

Quantification of collapsed growth cones revealed that the treatment of DRGs with Sema 3A in DM or with Sema 3A-free DM collapsed the growth cones up to $79 \%$ and $12 \%$, respectively (Figure $3, \mathrm{P}<0.001$, compared to Sema 3A supplemented DM). Treatment of DRGs with Sema 3A-supplemented CM of non-transduced and Lv-SFFV-eGFP vector transduced HEK293T cells and has led to a collapse of approximately $47 \%$ and $43 \%$ of growth cones, respectively, with no significant difference between these two groups, as expected (Figure 3, $\mathrm{P}>0.05$ ). However, when cultured DRGs were treated with CM containing Sema 3A and msNRP1, 27.7\% of growth cones were collapsed. There were significant differences between groups treated with $\mathrm{CM}$ of nontransduced $(\mathrm{P}<0.01)$ or Lv-SFFV-eGFP vector transduced cells $(\mathrm{P}<0.05)$ (Figure 3$)$. On the other hand, the difference in the collapse of growth cones between LvSFFV-msNRP1 vector transduced HEK293T CM, and Sema 3A-free DM groups were not significant $(\mathrm{P}=0.07)$. As a result, the present study suggests that endogenous SNRP1 protein in mice can inhibit the collapse activity of Sema $3 \mathrm{~A}$ in vitro. 


\section{Discussion}

We cloned a soluble form of Neuropilin 1 (SNRP1) containing a1/a1-b1/b2 domains. HEK293T cells were transduced with the lentivirus containing sNRP1. ELISA results showed that the SNRP1 was expressed in HEK293T cells. A conditioned medium of transduced HEK293T cells was used to investigate if it can bind with Sema 3A and inhibit Sema3A-induced growth cone collapse. The results showed that the conditioned medium containing SNRP1 could reduce the growth cone collapse of Sema 3A.

Neuropilins are important receptors that participate in several signaling pathways, including class 3 semaphorins and VEGF pathways (Chen, Chedotal, He, Goodman, \& Tessier-Lavigne, 1997; Gluzman-Poltorak, Cohen, Herzog, \& Neufeld, 2000; Kolodkin et al., 1997; Mamluk et al., 2002). NRPs are transmembrane ligandbinding receptors. When NRPs are bound with their ligand, they bind to their co-receptors responsible for signal transduction. One of these co-receptors is Plexin (PLXN), recruited to NRPs when the ligand is Sema 3A (Soker, Miao, Nomi, Takashima, \& Klagsbrun, 2002). Overall, NRPs play a part in various biological processes such as angiogenesis, tumor development, and neuronal guidance during nervous system development or regeneration (cited in Pasterkamp \& Giger, 2009).

Researchers have previously cloned and produced recombinant NRP or NRP inhibitors to prevent transmembrane NRP signal transduction. In 1999, Renzi et al. showed that antibodies against NRP1 could inhibit NRP1 downstream signaling (Renzi, Feiner, Koppel, \& Raper,1999). Regarding VEGF-NRP interaction, various studies have shown that natural soluble forms of NRP act as ligand-scavengers and antagonized VEGF in vivo (Gagnon et al., 2000; Rossignol et al., 2000). We used a simple yet functional approach to prevent Sema 3A inhibitory effects on growth cones. We cloned only the a1/ a2-b1/b2 fragment, an alternatively spliced natural form of NRP. Then, HEK293T cells were transduced, and the conditioned medium was concentrated. ELISA results on the conditioned medium confirmed the successful expression of the cloned fragment of NRP1 (soluble NRP1).

Other researchers have explored non-NRP compounds to inhibit Sema 3A-NRP1 interaction. Montolio et al. showed that SICHI peptoid could inhibit Sema 3A binding to NRP1 (Montolio et al., 2009). Cackowski et al. also found two soluble forms of NRP1 (sIIINRP1 and sIVNRP1). They showed that these sNRP1s could an- tagonize Sema 3A- and VEGF-165-dependent cellular activities (Cackowski et al., 2004).

Studies show that membrane NRP1 is a receptor for Sema $3 \mathrm{~A}$. NRP1 ${ }^{+}$cells migrate in response to locally secreted Sema 3A. Dejda et al. showed that genetic ablation of NRP1 and therapeutics that trap NRP1 could reduce immune cell recruitment to lesion site and neovascularization in retinopathies (Dejda et al., 2014). In MS lesions, the level of Sema 3A increases, recruits, and activates $\mathrm{NRP}^{+}$cells to the lesion site, resulting in MS complications (Williams et al., 2007). We hypothesized that the collapse activity of Sema 3A could be inhibited by neutralizing its binding to membrane NRP1. Thus, we used a soluble form of NRP1, which competitively binds to Sema 3A and prevents it from binding to cell surface NRP1. We demonstrated that NRP1-containing conditioned media could effectively bind Sema $3 \mathrm{~A}$ and prevent growth cone collapse of DRGs.

The results suggest that soluble NRP1 can bind Sema 3A and inhibit Sema 3A-dependent NRP1 signaling. Therefore, sNRP1 is probably effective in neuronal remyelination in MS lesions. However, mouse models of MS are needed to evaluate the effects of sNRP1 systemic administration.

\section{Ethical Considerations}

\section{Compliance with ethical guidelines}

All procedures of the study have been approved by the Ethics Committee of Zanjan University of Medical Sciences, Zanjan, Iran (ZUMS.REC.1394.264), and have been confirmed to the Declaration of Helsinki.

\section{Funding}

This study was supported by Cancer Gene Therapy Research Center, Zanjan University of Medical Sciences, Zanjan, Iran (Grant No.: A-12-65-8).

\section{Authors' contributions}

Conceptualization and supervision: Keivan Nedaei, Mehdi Totonchi, and Ali Reza Biglari; Methodology: Keivan Nedaei and Mahdi Hesaraki; Investigation, writing - original draft, and writing - review \& editing: Keivan Nedaei; Data collection and data analysis: Saeideh Mazloomzadeh; Funding acquisition and resources: Alireza Biglari and Mehdi Totonchi. 


\section{Conflict of interest}

The authors declared no conflict of interest.

\section{Acknowledgments}

The authors would like to thank Dr. Tina Shahani for technical supports and Dr. Vahid Kia for revising the English version manuscript.

\section{References}

Cackowski, F. C., Xu, L., Hu, B., \& Cheng, S. Y. (2004). Identification of two novel alternatively spliced Neuropilin-1 isoforms. Genomics, 84(1), 82-94. [DOI:10.1016/j.ygeno.2004.02.001] [PMID] [PMCID]

Chen, H., Chedotal, A., He, Z., Goodman, C. S., \& Tessier-Lavigne, M. (1997). Neuropilin-2, a novel member of the neuropilin family, is a high affinity receptor for the semaphorins Sema E and Sema IV but not Sema III. Neuron, 19(3), 547-59. [DOI:10.1016/S0896-6273(00)80371-2]

Costa, C., Martínez-Sáez, E., Gutiérrez-Franco, A., Eixarch, H., Castro, Z., \& Ortega-Aznar, A., et al. (2015). Expression of semaphorin 3A, semaphorin 7A and their receptors in multiple sclerosis lesions. Multiple Sclerosis Journal, 21(13), 1632-43. [DOI:10.1177/1352458515599848] [PMID]

De Winter, F., Oudega, M., Lankhorst, A. J., Hamers, F. P., Blits, B., \& Ruitenberg, M. J., et al. (2002). Injury-induced class 3 semaphorin expression in the rat spinal cord. Experimental Neurology, 175(1), 61-75. [DOI:10.1006/exnr.2002.7884] [PMID]

Dejda, A., Mawambo, G., Cerani, A., Miloudi, K., Shao, Z., \& Daudelin, J. F., et al. (2014). Neuropilin-1 mediates myeloid cell chemoattraction and influences retinal neuroimmune crosstalk. Journal of Clinical Investigation, 124(11), 4807-22. [DOI:10.1172/JCI76492] [PMID] [PMCID]

Franklin, R. J. (2008). Remyelination in the CNS: From biology to therapy. Nature Reviews Neuroscience, 9(11), 839. [DOI:10.1038/ nrn2480] [PMID]

Gagnon, M. L., Bielenberg, D. R., Gechtman, Z. E., Miao, H Q., Takashima, S., \& Soker, S., et al. (2000). Identification of a natural soluble neuropilin-1 that binds vascular endothelial growth factor: In vivo expression and antitumor activity. Proceedings of the National Academy of Sciences, 97(6), 2573-8. [DOI:10.1073/pnas.040337597] [PMID] [PMCID]

Gluzman-Poltorak, Z., Cohen, T., Herzog, Y., \& Neufeld, G. (2000). Neuropilin-2 is a receptor for the Vascular Endothelial Growth Factor (VEGF) forms VEGF-145 and VEGF-165 [corrected]. Journal of Biological Chemistry, 275(24), 18040-5. [DOI:10.1074/jbc.M909259199] [PMID]

Good, P. F., Alapat, D., Hsu, A., Chu, C., Perl, D., \& Wen, X., et al. (2004). A role for semaphorin $3 \mathrm{~A}$ signaling in the degeneration of hippocampal neurons during Alzheimer's disease. Journal of Neurochemistry, 91(3), 716-36. [DOI:10.1111/j.14714159.2004.02766.x] [PMID]
Joyal, J. S., Sitaras, N., Binet, F., Rivera, J. C., Stahl, A., \& Zaniolo, K., et al. (2011). Ischemic neurons prevent vascular regeneration of neural tissue by secreting semaphorin 3A. Blood, The Journal of the American Society of Hematology, 117(22), 6024-35. [DOI:10.1182/ blood-2010-10-311589] [PMID] [PMCID]

Kapfhammer, J. P., Xu, H., \& Raper, J. A. (2007). The detection and quantification of growth cone collapsing activities. Nature Protocols, 2(8), 2005. [DOI:10.1038/nprot.2007.295] [PMID]

Kitsukawa, T., Shimizu, M., Sanbo, M., Hirata, T., Taniguchi, M., \& Bekku, Y., et al. (1997). Neuropilin-semaphorin III/D-mediated chemorepulsive signals play a crucial role in peripheral nerve projection in mice. Neuron, 19(5), 995-1005. [DOI:10.1016/ S0896-6273(00)80392-X]

Kitsukawa, T., Shimono, A., Kawakami, A., Kondoh, H., \& Fujisawa, H. (1995). Overexpression of a membrane protein, neuropilin, in chimeric mice causes anomalies in the cardiovascular system, nervous system and limbs. Development, 121(12), 430918. [DOI:10.1242/dev.121.12.4309]

Kolodkin, A. L., Levengood, D. V., Rowe, E. G., Tai, Y. T., Giger, R. J., \& Ginty, D. D. (1997). Neuropilin is a semaphorin III receptor. Cell, 90(4), 753-62. [DOI:10.1016/S0092-8674(00)80535-8]

Körner, S., Böselt, S., Wichmann, K., Thau-Habermann, N., Zapf, A., \& Knippenberg, S., et al. (2016). The axon guidance protein semaphorin $3 \mathrm{~A}$ is increased in the motor cortex of patients with amyotrophic lateral sclerosis. Journal of Neuropathology $\mathcal{E} E x$ perimental Neurology, 75(4), 326-33. [DOI:10.1093/jnen/nlw003] [PMID]

Le Guelte, A., Galan-Moya, E. M., Dwyer, J., Treps, L., Kettler, G., \& Hebda, J. K., et al. (2012). Semaphorin 3A elevates endothelial cell permeability through PP2A inactivation. Journal of cell Science, 125(17), 4137-46. [DOI:10.1242/jcs.108282] [PMID]

Luo, Y., Raible, D., \& Raper, J. A. (1993). Collapsin: A protein in brain that induces the collapse and paralysis of neuronal growth cones. Cell, 75(2), 217-27. [DOI:10.1016/0092-8674(93)80064-L]

Mamluk, R., Gechtman, Z., Kutcher, M. E., Gasiunas, N., Gallagher, J., \& Klagsbrun, M. (2002). Neuropilin-1 binds vascular endothelial growth factor 165, placenta growth factor-2, and heparin via its b1b2 domain. Journal of Biological Chemistry, 277(27), 24818-25. [DOI:10.1074/jbc.M200730200] [PMID]

Mamluk, R., Klagsbrun, M., Detmar, M., \& Bielenberg, D. R. (2005). Soluble neuropilin targeted to the skin inhibits vascular permeability. Angiogenesis, 8(3), 217-27. [DOI:10.1007/s10456005-9009-6] [PMID]

Miao, H. Q., Soker, S., Feiner, L., Alonso, J. L., Raper, J. A., \& Klagsbrun, M. (1999). Neuropilin-1 mediates collapsin-1/semaphorin III inhibition of endothelial cell motility: Functional competition of collapsin-1 and vascular endothelial growth factor-165. The Journal of Cell Biology, 146(1), 233-42. [DOI:10.1083/ jcb.146.999.233] [PMID] [PMCID]

Montolio, M., Messeguer, J., Masip, I., Guijarro, P., Gavin, R., \& Antonio Del Rio, J., et al. (2009). A semaphorin 3A inhibitor blocks axonal chemorepulsion and enhances axon regeneration. Chemistry \& Biology, 16(7), 691-701. [DOI:10.1016/j.chembiol.2009.05.006] [PMID]

Panigrahy, D., Adini, I., Mamluk, R., Levonyak, N., Bruns, C. J., \& D'Amore, P. A., et al. (2014). Regulation of soluble neuropilin 1, an endogenous angiogenesis inhibitor, in liver development and regeneration. Pathology, 46(5), 416-23. [DOI:10.1097/ PAT.0000000000000121] [PMID] [PMCID] 
Pasterkamp, R. J., \& Giger, R. J. (2009). Semaphorin function in neural plasticity and disease. Current Opinion in Neurobiology, 19(3), 263-74. [DOI:10.1016/j.conb.2009.06.001] [PMID] [PMCID]

Piaton, G., Aigrot, M. S., Williams, A., Moyon, S., Tepavcevic, V., \& Moutkine, I., et al. (2011). Class 3 semaphorins influence oligodendrocyte precursor recruitment and remyelination in adult central nervous system. Brain, 134(Pt 4), 1156-67. [DOI:10.1093/brain/awr022] [PMID]

Renzi, M. J., Feiner, L., Koppel, A. M., \& Raper, J. A. (1999). A dominant negative receptor for specific secreted semaphorins is generated by deleting an extracellular domain from neuropilin-1. Journal of Neuroscience, 19(18), 7870-80. [DOI:10.1523/ JNEUROSCI.19-18-07870.1999] [PMID] [PMCID]

Rossignol, M., Gagnon, M. L., \& Klagsbrun, M. (2000). Genomic organization of human neuropilin-1 and neuropilin-2 genes: identification and distribution of splice variants and soluble isoforms. Genomics, 70(2), 211-22. [DOI:10.1006/ geno.2000.6381] [PMID]

Soker, S., Miao, H. Q., Nomi, M., Takashima, S., \& Klagsbrun, M. (2002). VEGF165 mediates formation of complexes containing VEGFR-2 and neuropilin-1 that enhance VEGF165-receptor binding. Journal of Cellular Biochemistry, 85(2), 357-68. [DOI:10.1002/jcb.10140] [PMID]

Syed, Y. A., Hand, E., Möbius, W., Zhao, C., Hofer, M., \& Nave, K. A., et al. (2011). Inhibition of CNS remyelination by the presence of semaphorin 3A. Journal of Neuroscience, 31(10), 3719-28. [DOI:10.1523/JNEUROSCI.4930-10.2011] [PMID] [PMCID]

Wang, L., \& Marquardt, T. (2012). Direct live monitoring of heterotypic axon-axon interactions in vitro. Nature Protocols, 7(2), 351. [DOI:10.1038/nprot.2011.442] [PMID]

Weber, K., Bartsch, U., Stockinwg, C., \& Fehse, B. (2008). A multicolor panel of novel Lentiviral "Gene Ontology"(LeGO) vectors for functional gene analysis. Molecular Therapy, 16(4), 698-706. [DOI:10.1038/mt.2008.6] [PMID]

Williams, A., Piaton, G., Aigrot, M.-S., Belhadi, A., Théaudin, M., \& Petermann, F., et al. (2007). Semaphorin 3A and 3F: key players in myelin repair in multiple sclerosis? Brain, 130(10), 2554-65. [DOI:10.1093/brain/awm202] [PMID] 